\title{
Weak Lensing Minima and Peaks: Cosmological Constraints and the Impact of Baryons
}

\author{
William R. Coulton, ${ }^{1 \star}$ Jia Liu, ${ }^{2,3,4}$ Ian G. McCarthy ${ }^{5}$ and Ken Osato ${ }^{6}$ \\ ${ }^{1}$ Institute of Astronomy and Kavli Institute for Cosmology Cambridge, Madingley Road, Cambridge, CB3 OHA, UK \\ ${ }^{2}$ Berkeley Center for Cosmological Physics, University of California, Berkeley, CA 94720, USA \\ ${ }^{3}$ Lawrence Berkeley National Laboratory, 1 Cyclotron Road, Berkeley, CA 93720, USA \\ ${ }^{4}$ Department of Astrophysical Sciences, Princeton University, Peyton Hall, Princeton, NJ 08544, USA \\ ${ }^{5}$ Astrophysics Research Institute, Liverpool John Moores University, 146 Brownlow Hill, Liverpool, L3 5RF, UK \\ ${ }^{6}$ Institut d'Astrophysique de Paris, Sorbonne Université, CNRS, UMR 7095, 98bis boulevard Arago, F-75014 Paris, France
}

\begin{abstract}
We present a novel statistic to extract cosmological information in weak lensing data: the lensing minima. We also investigate the effect of baryons on cosmological constraints from peak and minimum counts. Using the MassiveNuS simulations, we find that lensing minima are sensitive to non-Gaussian cosmological information and are complementary to the lensing power spectrum and peak counts. For an LSST-like survey, we obtain $95 \%$ credible intervals from a combination of lensing minima and peaks that are significantly stronger than from the power spectrum alone, by $44 \%, 11 \%$, and $63 \%$ for the neutrino mass sum $\sum m_{v}$, matter density $\Omega_{m}$, and amplitude of fluctuation $A_{s}$, respectively. We explore the effect of baryonic processes on lensing minima and peaks using the hydrodynamical simulations BAHAMAS and Osato15. We find that ignoring baryonic effects would lead to strong $(\approx 4 \sigma)$ biases in inferences from peak counts, but negligible $(\approx 0.5 \sigma)$ for minimum counts, suggesting lensing minima are a potentially more robust tool against baryonic effects. Finally, we demonstrate that the biases can in principle be mitigated without significantly degrading cosmological constraints when we model and marginalize the baryonic effects.
\end{abstract}

Key words: Gravitational lensing: weak, neutrinos, methods: statistical, galaxies: haloes

\section{INTRODUCTION}

Weak gravitational lensing of background galaxies probes the integrated matter fluctuations along the line-of-sight. It is sensitive to fundamental physics such as the nature of dark energy and the total mass of neutrinos (see a recent review by Kilbinger 2015). Recently, pioneering weak lensing surveys achieved high precision measurements, leading to competitive constraints on cosmology (Heymans et al. 2012; DES Collaboration et al. 2017; Hildebrandt et al. 2017; Hikage et al. 2019). At present, results from weak lensing measurements have been primarily driven by two-point (or second-order) measurements, such as the two-point correlation function or its Fourier transform, the power spectrum. However, nonlinear structure growth on scales smaller than a few $\times 10 \mathrm{Mpc}$ moves a significant portion of cosmological information from second-order to higher-order statistics. As the next generation weak lensing surveys are coming online in the next decade, such as the $\mathrm{LSST}^{1}$ (LSST Sci-

\footnotetext{
^ E-mail: wcoulton@ ast.cam.ac.uk

1 Large Synoptic Survey Telescope: http://www. lsst.org
}

ence Collaboration et al. 2009a), WFIRST ${ }^{2}$, and Euclid $^{3}$, we will be probing deep into the nonlinear regime and two-point statistics will no longer be adequate.

To access this information we need to utilize higher-order, nonGaussian statistics. Naturally, one would consider the next-order term, the three-point correlation function (or its Fourier transform, the bispectrum) (Takada \& Jain 2003; Vafaei et al. 2010; Fu et al. 2014; Coulton et al. 2019). However, three-point functions can be computationally expensive, due to the large number of possible triangle shapes in a typical lensing dataset, and have large, complex covariance matrices. Further, by definition it only accesses third-order information and is therefore insensitive to information in fourth- and higher-order moments. Therefore, weak lensing summary statistics, which can be easier to measure and also contain information of all orders, have been proposed as simpler alternatives, such as the peak counts (Jain \& Van Waerbeke 2000; Marian et al. 2009; Maturi et al. 2010; Yang et al. 2011; Marian et al. 2013; Liu et al. 2015, 2014; Lin \& Kilbinger 2015a,b; Kacprzak et al. 2016; Peel et al. 2018;

\footnotetext{
2 Wide-Field Infrared Survey Telescope: http://wfirst.gsfc.nasa. gov

3 Euclid: http://sci.esa.int/euclid
} 
Shan et al. 2018; Martinet et al. 2018; Li et al. 2019), Minkowski functionals (Kratochvil et al. 2012; Shirasaki \& Yoshida 2014; Petri et al. 2013, 2015; Marques et al. 2019), clipped field statistics (Giblin et al. 2018), and higher-order moments (Bernardeau et al. 1997; Hui 1999; Van Waerbeke et al. 2001; Takada \& Jain 2002; Zaldarriaga \& Scoccimarro 2003; Kilbinger \& Schneider 2005; Petri et al. 2015; Peel et al. 2018).

In this paper, we propose a new probe of non-Gaussian information - weak lensing minima. The name encapsulates the nature of the statistic - the number counts of local minima in a (typically smoothed) lensing convergence map as a function of their depth. Computationally, they are the pixels with values smaller than their surrounding pixels. Our motivation to investigate lensing minima is threefold. First, they probe the emptiest regions (voids) in our universe and hence are complementary to the well-investigated lensing peaks, which are typically associated with massive halos. Second, the baryonic effects are expected to impact voids differently than overdense regions, leading us to postulate that baryonic physics, one of the most worrisome lensing systematics, would impact lensing minima differently than other non-Gaussian statistics and may in turn help constrain baryonic physics. Finally, lensing minima are a particularly simple non-Gaussian statistic, as they are easily computed from observational data and can be modeled using existing weak lensing simulations built for other lensing statistics.

Lensing signals around underdense regions in the universe have been previously studied both theoretically and with observational data. For example, Sánchez et al. (2017) used DES redMaGiC galaxies to identify voids and found the stacked lensing signal around them to be negative at a $4 \sigma$ level (also see Melchior et al. 2014; Clampitt \& Jain 2015; Gruen et al. 2016). The lensing profile around underdense regions has also been a topic of interest for modified gravity, as dark energy is more prominent in void regions (Barreira et al. 2015, 2017; Baker et al. 2018; Paillas et al. 2019). Void lensing can be more complicated than cluster lensing, as voids may have irregular shapes and the lensing signal depends on the void identification scheme. For example, Davies et al. (2018) found a significantly higher tangential shear signal around voids identified using lensing peaks, compared to those found using galaxies.

Our method has the advantage of simplicity — lensing minima do not require any void tracer, such as halos or lensing peaks as needed in previous works. While we expect the lensing minima to be mostly associated with void regions (i.e. with negative lensing signal), we find that a small number of them have positive values and hence also have imprints from slightly overdense regions. In this paper, we show that lensing minima are sensitive to cosmology and forecast the cosmological constraints for an LSST-like survey. We compare our results to the power spectrum, a traditional lensing measurement, and the peak counts, a better-studied lensing nonGaussian statistic.

Measurements of peak and minimum counts can be sensitive to very small scales. As such, they could be impacted by the effects of baryons. Baryonic processes result in a suppression of the matter power spectrum at scales $k \approx 1 h \mathrm{Mpc}^{-1}$, mainly due to feedback processes of active galactic nuclei (AGN) and supernova explosions, and an enhancement of the very small scale power spectrum $k \gtrsim 10 h \mathrm{Mpc}^{-1}$ due to baryonic cooling (van Daalen et al. 2011; Schaye et al. 2015; McCarthy et al. 2017; Nelson et al. 2019). These processes may impact peak and minimum counts by altering the depth of the potential wells. The impact of baryonic processes on peak counts has been studied in previous works (e.g. Yang et al. 2013; Osato et al. 2015; Fong et al. 2019; Weiss et al. 2019). Here, we extend the study to cosmological parameter constraints, using hydrodynamical simulations. We will also present the first study of the impact of baryons on lensing minima.

The paper is organized as follows. First, in Section 2, we provide an overview of the simulations used in this work. In Section 3, we explore the properties of lensing minima measured in our simulated lensing convergence maps and compare them with power spectrum and peak counts. Next, in Section 4, we explore the cosmological constraining power of lensing minima for an LSST-like survey and compare the constraints with those from the power spectrum and peak counts. We also show that baryonic physics have different impact on minima compared to the other two statistics in Section 5. Finally, we conclude in Section 6.

\section{SIMULATIONS}

To understand the cosmological power of lensing minima and their sensitivity to baryonic physics, we use two types of simulations - a set of dark-matter only simulations that model a grid of cosmological parameters, the Cosmological Massive Neutrino Simulations (MassiveNuS Liu et al. 2018) and two sets of hydrodynamical simulations - the BAryons and HAloes of MAssive Systems (BAHAMAS McCarthy et al. 2017, 2018) and the set used in Osato et al. (2015) (0sato15). Here we briefly describe the key aspects of these simulations relevant to this work, and refer the readers to the code papers for more details.

MassiveNuS consists of 101 flat $\Lambda$ cold dark matter $(\Lambda \mathrm{CDM})$ $N$-body simulations, with three varied parameters: the neutrino mass sum $\sum m_{v}$, the total matter density $\Omega_{m}$, and the amplitude of primordial fluctuation $A_{S}$, covering the parameter ranges $\sum m_{v}=[0,0.62] \mathrm{eV}, \Omega_{m}=[0.18,0.42]$, and $10^{9} A_{s}=[1.29,2.91]$. The simulations have a box size of $512 h^{-1} \mathrm{Mpc}$ and $1024^{3} \mathrm{CDM}$ particles, accurately capturing structure growth at $k \lesssim 10 h \mathrm{Mpc}^{-1}$. Massive neutrinos are treated using linear perturbation theory and their clustering is sourced by the full nonlinear matter density (AliHaïmoud \& Bird 2013; Bird et al. 2018), and the resulting accuracy of the total matter power spectrum is tested to agree with particle neutrino simulations to within $0.2 \%$ for $\sum m_{v}<0.6 \mathrm{eV}$. In this work we choose one simulation with cosmological parameters $\sum m_{v}=0.1 \mathrm{eV}, \Omega_{m}=0.3$, and $10^{9} A_{s}=2.1$ as our fiducial cosmology. Weak lensing convergence maps are available for five delta-function source redshifts $z_{s}=0.5,1.0,1.5,2.0,2.5$ with 10,000 realizations generated per model per source redshift using the ray-tracing code LensTools (Petri 2016) ${ }^{4}$. Maps have $512^{2}$ pixels and are $3.5^{2}=12.25 \mathrm{deg}^{2}$ in size. The maps at different source redshifts are ray-traced through the same large-scale structure, and hence are properly correlated.

To study the effect of baryons, we use two sets of hydrodynamical simulations. The BAHAMAS simulations have a box size of $400 h^{-1} \mathrm{Mpc}$ and $2 \times 1024^{3}$ particles. The simulations were run with the Gadget-3 TreePM SPH code, which was modified to include subgrid prescriptions for metal-dependent radiative cooling, star formation, stellar and chemical evolution, black hole formation and merging, and stellar and AGN feedback, originally developed for the OWLS project (Schaye et al. 2010). For the fiducial BAHAMAS model, the parameters characterising the efficiencies of stellar and AGN feedback were adjusted to approximately reproduce the observed present-day galaxy stellar mass function (above $10^{10} \mathrm{M}_{\odot}$ ) and the amplitude of the local hot gas fraction-halo mass relation,

\footnotetext{
4 https://pypi.python.org/pypi/lenstools
} 


\begin{tabular}{lccccc}
\hline$z_{s}$ & 0.5 & 1.0 & 1.5 & 2.0 & 2.5 \\
\hline $\bar{n}_{\text {gal }}\left(\operatorname{arcmin}^{-2}\right)$ & 8.83 & 13.25 & 11.15 & 7.36 & 4.26 \\
\hline
\end{tabular}

Table 1. The projected source counts per $\operatorname{arcmin}^{2}$ used in our tomography analysis. For the single redshift $z_{s}=1$ analysis, we use $\bar{n}_{\text {gal }}=$ $44.8 \operatorname{arcmin}^{-2}$.

as inferred from high-resolution X-ray observations. In addition to the fiducial BAHAMAS simulation with a feedback model designed to best match these observations, we also include two additional simulations where the AGN heating temperature is raised and lowered by 0.2 dex, hereafter "high AGN" and "low AGN". Varying the heating temperature in this way retains a good match to the galaxy stellar mass function but changes the gas fractions of haloes (and therefore their lensing signals) so that the simulations skirt the upper and lower bounds of the observed gas fractions. We generate 10,000 convergence maps from 25 independent light cones (McCarthy et al. 2018), using a similar technique as in the MassiveNuS.

The Osato15 simulations have a box size of $240 h^{-1} \mathrm{Mpc}$ and $2 \times 512^{3}$ particles. This set of simulations employs the recipe of galaxy formation physics developed in Okamoto et al. (2014), where basic baryonic processes, e.g., star formation and radiative cooling, are implemented as the subgrid model. In this model, formation and evolution of black holes is not fully traced but an ad-hoc modeling is adopted to mimic the feedback effect. At each time step, the velocity dispersion within a halo is evaluated. If the velocity dispersion exceeds the threshold value, radiative cooling within the halo is manually stopped. Thus, further star formation is suppressed. 100 convergence maps are generated for both the dark matter-only simulations and the hydrodynamical runs (referred to as "FE" in the original paper) based on the ray-tracing technique.

Both sets of hydrodynamical simulations have parallel dark matter-only and hydrodynamical runs with the same initial conditions, at the WMAP 9-yr cosmology with $\left\{\Omega_{m}, \Omega_{b}, \Omega_{\Lambda}, \sigma_{8}, n_{s}, h\right\}=$ $\{0.2793,0.0463,0.7207,0.821,0.972,0.700\}$ (Hinshaw et al. 2013). The main difference between these two simulation suites is the implementation of AGN feedback. For BAHAMAS, the modeling of black holes is based on Booth \& Schaye (2009), where AGN feedback is modelled by thermally coupling a fraction of the restmass energy of the accreted gas into the surrounding medium, but for Osato2015 star formation is simply shut down for halos with large velocity dispersion. The latter model leads to weaker feedback than observations and smaller amounts of ejected gas (which has been found by van Daalen et al. (2019) to be strongly correlated with the effects of baryons on the power spectrum). By including two sets of hydrodynamical simulations, we demonstrate the differences among existing feedback models and hence stress the importance of careful modeling of baryonic effects.

To create LSST-like mocks, we follow the photometric redshift distribution and galaxy number density estimated in the LSST Science Book (LSST Science Collaboration et al. 2009b). We use the number density for each source redshift in Table 1, which is obtained by integrating the LSST source distribution function (Eq 3.8 of LSST Science Collaboration et al. (2009b)) with top hat windows functions of width $\Delta z=0.5$ centered on $z=0.5,1,1.5,2,2.5$ .We then add Gaussian noise to the simulated convergence maps with a variance $V_{\text {noise }}$ of

$V_{\text {noise }}=\frac{\sigma_{s}^{2}}{n_{g}}$

where $\sigma_{S}=0.3$ is the intrinsic shape noise and $n_{g}$ is the galaxy number density in Table 1. The shape noise leads to a large number of false minima. Therefore, before measuring the minima, we smooth our maps with a Gaussian filter of $\theta_{G}=2$ arcmin. We measure the counts of minima as a function of the depth, normalized by the standard deviation of the smoothed noise-only maps $\sigma_{n}$, where

$\sigma_{n}^{2}=\frac{\sigma_{s}^{2}}{4 \log (2) \pi \theta_{G}^{2} n_{g}}$.

We study the cosmological constraints from minima with two different redshift settings - the tomographic setting as shown in Table 1 and a single redshift distribution assuming all galaxies are at $z_{s}=1$. Both settings have the same total number of galaxies per $\operatorname{arcmin}^{2}$. Our study of baryonic physics is only applied to the single source redshift setting.

\section{WEAK LENSING MINIMA}

We identify lensing minima in our simulated convergence maps as pixels with lower values than their 8 neighbours. These minima are then binned by their depth, forming the minimum counts. The convergence maps are smoothed first, to reduce the impact of galaxy shape noise. The dependence of our results on smoothing scales is explored in Appendix A. This procedure is analogous to lensing peaks, which are identified as those with higher values than their 8 neighbours and are then similarly binned by height.

\subsection{Non-Gaussian information in lensing minima}

In Fig. 1, we show the minimum counts from the noise-free maps at the MassiveNuS simulations at a massless neutrino cosmology ( $\sum m_{v}=0.0 \mathrm{eV}, \Omega_{m}=0.3$, and $10^{9} A_{s}=2.1$ ), for five source redshifts. For comparison, we also show the peak counts. As expected, the minima primarily occur in underdense regions with negative $\kappa$ values. Compared to the shape of peak counts, the distribution of the minimum counts is slightly narrower and more symmetric. Note these effects are stronger than Fig. 1 implies as the $x$-axis range is smaller for the minimum counts. While the high $\kappa$ tails in the peak counts are formed due to the highly nonlinear regions, very long negative $\kappa$ tails in the minimum counts are absent because of a minimum possible $\kappa$ due to the density contrast limit $\delta=\rho / \bar{\rho}-1 \in[-1,+\infty)$.

We next investigate if the non-Gaussian signals in the noisefree case remain when we consider mock observations including the impact of galaxy shape noise. In Fig. 2, we show the number counts for maps with galaxy noises expected from LSST. The errorbars are computed by measuring the variance of each bin using 10,000 simulated patches and then scaling the spread by $f_{\text {sky }}$ to represent the LSST area. In addition, we show the number counts from Gaussian random fields (GRF) that have the same power spectra as the simulated maps (Bond \& Efstathiou 1987). For peak counts, we find more peaks at the high $\kappa$ tails in the simulations than in the GRFs, as the nonlinear growth in the simulations leads to non-Gaussianity. This is consistent with previous works on peak counts (Yang et al. 2011; Li et al. 2019). For minimum counts, the non-Gaussian signatures are more prominent in the negative $\kappa$ tails, with less minima in the simulations than in the GRFs.

It is clear that lensing minima contain rich non-Gaussian information that is beyond the power spectrum, even when galaxy noise is included. We next explore their sensitivity to cosmological parameters, with a focus on the sum of neutrino masses. 

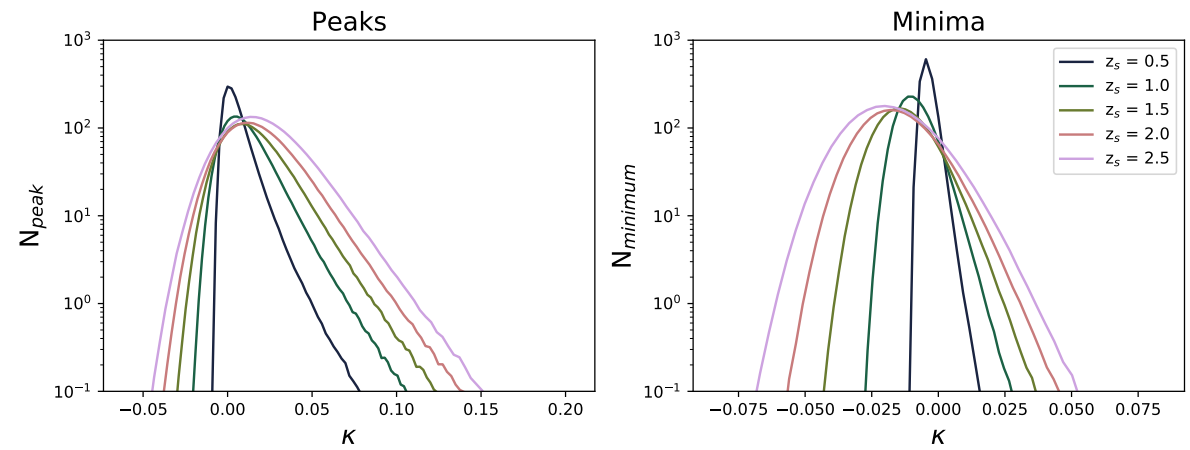

Figure 1. Weak lensing peak counts (left) and minimum counts (right) as a function of the convergence $\kappa$ height/depth from the MassiveNuS noise-free simulations, for five source redshifts.
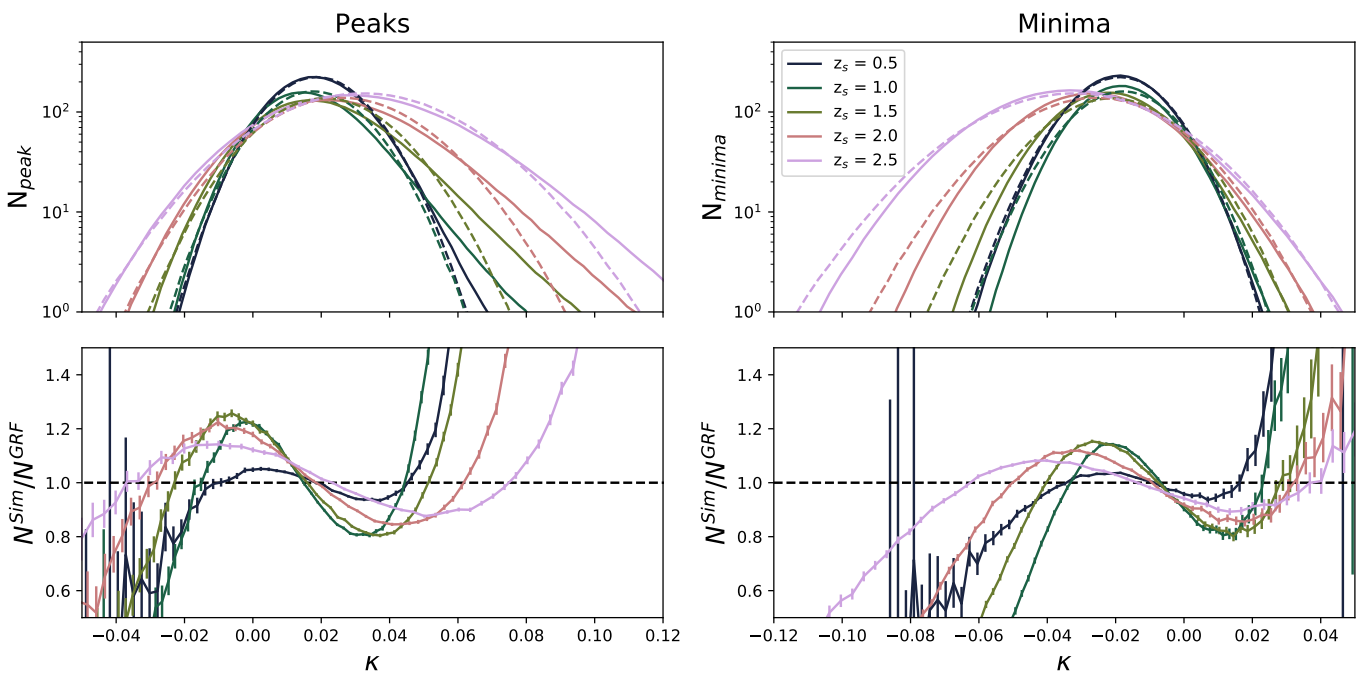

Figure 2. Upper panels: Peak counts (left) and minimum counts (right) as a function of the convergence $\kappa$ height/depth from the MassiveNuS simulation (solid lines) and from Gaussian random fields (GRFs, dashed lines). Lower panels: ratios of the peak and minimum counts obtained from simulations to that of the GRFs. Galaxy noise is included. The error bars are for an LSST-like survey that covers 20,000 $\mathrm{deg}^{2}$.

\subsection{Effect of neutrino mass}

Massive neutrinos suppress the growth of structures below the free streaming scale (Lesgourgues \& Pastor 2006):

$k_{F}=0.0072\left(\frac{\sum m_{v}}{0.1 \mathrm{eV}}\right)^{\frac{1}{2}}\left(\frac{\Omega_{m}}{0.315}\right)^{\frac{1}{2}} h \mathrm{Mpc}^{-1}$.

Thus the scales typically probed by weak lensing analyses lie below the transition scale. For power spectrum measurements this makes it difficult to differentiate between massive neutrinos and different values of $A_{s}$ as, without the transition feature, massive neutrino suppression could be mimicked by a lower values $A_{s}$. This work explores whether minimum counts can weaken this degeneracy. We show in Fig. 3 the effect of massive neutrinos, $\Omega_{m}$, and $A_{s}$ on lensing minima, by comparing a new cosmology where we vary one parameter at a time to the fiducial massive neutrino cosmology ( $\sum m_{v}=0.1 \mathrm{eV}, \Omega_{m}=0.3,10^{9} A_{s}=2.1$ ). LSST noise is included.

When increasing the neutrino mass, we see a reduction of deep (very negative $\kappa$ ) minima. As neutrinos free stream from overdense regions, they "fill in" underdense regions, resulting in less-empty voids. Works by Massara et al. (2015); Kreisch et al. (2019) found that increasing neutrino mass reduces the number of large voids, which are necessary to create the deepest minima in lensing maps. Changes in $\Omega_{m}$ and $A_{s}$ have a similar effect, and hence can mimic the effects of neutrino mass. However, in the next section, we show that there are subtle differences in these curves that can help break the degeneracies.

For comparison we include peak counts in Fig. 3. Previously, Li et al. (2019) studied the impact of massive neutrinos on lensing peaks and found a reduction in high lensing peaks, consistent with the expectation that massive neutrinos suppress the formation of massive halos.

We also show the effect of baryons in Fig. 3. Here we use the fiducial AGN model and dark matter-only model from the BAHAMAS simulations. While baryonic feedback also seems to suppress deep minima, the zero-crossing points and shapes are different from those caused by cosmological parameters, for both minimum counts and peak counts. We discuss the implications in detail in Section 5.

\section{COSMOLOGICAL CONSTRAINTS}

To study the cosmological information in lensing minima, we use the full 101 cosmologies from the MassiveNuS simulation to build 

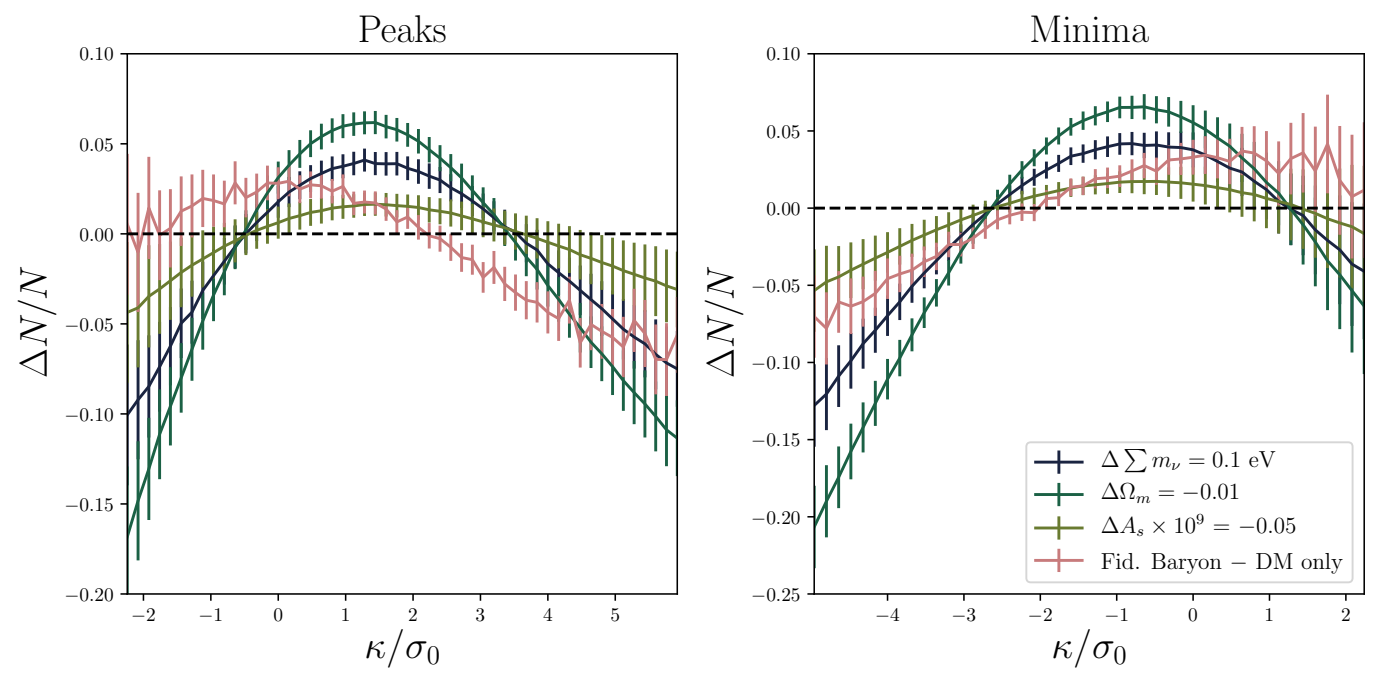

Figure 3. The fractional difference in peak counts (left) and minimum counts (right) due to variation of three cosmological parameters $\left(\sum m_{v}, \Omega_{m}, A_{s}\right)$ and baryons for simulations with LSST shape noise, for a single source redshift $z_{s}=1$. For the baryonic effects, we use the fiducial AGN feedback model and the dark matter-only model from the BAHAMAS simulations.

an emulator that models the statistics. We then use a Gaussian likelihood to estimate the credible regions for the minimum counts, peak counts, power spectrum, and the combination of the minima and peaks. For both minimum and peak counts, we use equallyspaced signal-to-noise ratio (SNR) bins with width $\triangle \mathrm{SNR}=0.16$ in the range SNR $=[-4.16,1.6]$ and $[-1.44,4.48]$ for minimum counts (36 bins) and peak counts (37 bins), respectively.

\subsection{Covariance matrix}

We explore the correlation between the minimum counts, peak counts, and power spectrum in Fig. 4, which is also a slice of the covariance matrix in our likelihood analysis. We use 10,000 map realizations at the fiducial cosmology to construct the covariance matrix. The covariance matrix includes bin-to-bin correlations, covariance across different redshifts and the covariance between different statistics. In the noise-free case, we see large off-diagonal terms for both minimum and peak counts, within their self-blocks. In addition, we also see strong correlations between the peak and minimum counts. In the noisy case, this correlation remains, albeit weaker.

\subsection{Likelihood}

We assume a Gaussian likelihood in our analysis,

$\ln \mathcal{L}=-\frac{1}{2} \sum_{i, j, X, Y}\left(\hat{S}_{i}^{X}-\bar{S}_{i}^{X}\right)\left(\Sigma_{S}^{X Y}\right)_{i j}^{-1}\left(\hat{S}_{j}^{Y}-\bar{S}_{j}^{Y}\right)+$ const.

where $S_{i}^{X}$ is the $i^{\text {th }}$ bin of the observable $S$ in redshift bin $X, \bar{S}^{X}$ is the mean value at the fiducial cosmology, and $\Sigma_{S}^{X Y}$ is the full covariance matrix.

The Gaussian likelihood is an approximation for the peak and minimum counts as they both follow Poisson distributions. However, since we only consider bins with average counts more than 1.5 for our $12.25 \mathrm{deg}^{2}$ maps, we expect the number counts for LSST $\left(\approx 2 \times 10^{4} \mathrm{deg}^{2}\right)$ will be sufficiently large to be approximated with a Gaussian distribution.
In order to evaluate the statistics at arbitrary cosmologies, we construct an emulator from the 101 simulated cosmologies using Gaussian Processes (Heitmann et al. 2009; Kwan et al. 2013, 2015; Heitmann et al. 2016; Nishimichi et al. 2018; McClintock et al. 2019), with the triaxial squared exponential kernel. We can then interpolate the statistics to any cosmology within the sample parameter ranges. We verify that the interpolation errors are significantly smaller than the measurement errors. Further details of our emulator are described in detail in Li et al. (2019); Coulton et al. (2019). The simulation sample parameter ranges are used as the limits of our flat priors,

$$
\begin{aligned}
P\left(\sum m_{v}\right) & = \begin{cases}\text { const., } & \text { if } 0 \mathrm{eV} \leq \sum m_{v} \leq 0.62 \mathrm{eV} \\
0, & \text { otherwise }\end{cases} \\
P\left(\Omega_{m}\right) & = \begin{cases}\text { const., } & \text { if } 0.18 \leq \Omega_{m} \leq 0.42 \\
0, & \text { otherwise }\end{cases} \\
P\left(A_{s}\right) & = \begin{cases}\text { const., } & \text { if } 1.29 \leq 10^{9} A_{s}<2.91 \\
0, & \text { otherwise. }\end{cases}
\end{aligned}
$$

We then sample the likelihood with Affine invariant Markov chain Monte-Carlo sampler emcee (Foreman-Mackey et al. 2013) and used the corner and ChainConsumer libraries (Foreman-Mackey 2016; Hinton 2016) to visualize our results. In this analysis we used 32 walkers started in ball around true cosmology. The burn in period was 1000 iterations (i.e. 32,000 samples) and for our constraints we ran for 10,000 iterations (320,000 samples). No thinning of the chains is performed. 10,000 iterations are more than 50 times auto-correlation time for all parameters. Thus, obtained chains are sufficiently converged.

\subsection{Parameter constraints}

In Fig. 5 we show the $95 \%$ credible regions on $\sum m_{v}, \Omega_{m}$, and $A_{s}$ from minimum counts for an LSST-like survey. Convergence maps are smoothed with a 2 arcmin Gaussian filter. For comparison, we also plot the constraints from the peak counts and power spectrum. For the power spectrum we use $\ell_{\max }=3000$. Similar to the peaks, 

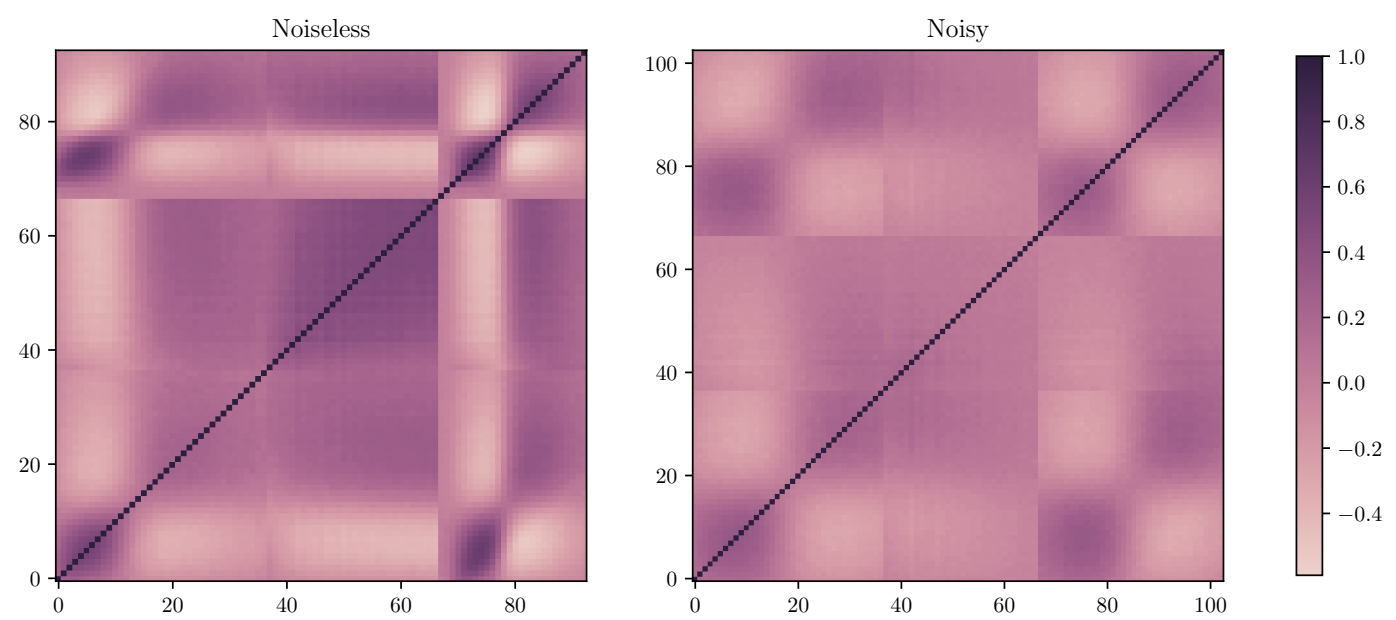

Figure 4. The full covariance matrix of the peak counts (bins 1-37), power spectrum (bins 38-67), and minimum counts (left: bins 68-93; right: bins 68-103) for $z_{s}=1.0$ from noiseless (left) and noisy (right) simulations. There are significantly more minima bins in the noisy case as the Gaussian noise broadens the distribution (see Figs. 1 and 2).

minima are stronger in constraining neutrino mass than the power spectrum. As the power spectrum optimally captures the information of a Gaussian field, the tighter constraints from minima imply that they are probing non-Gaussian information beyond the second order that is highly sensitive to cosmology. We show results from additional smoothing scales in Appendix A.

Finally, we explore the joint constraints combining minimum counts with peak counts. We find significant improvement with the joint constraint. This shows that minimum counts contains information independent of peak counts. The combined constraints from minimum and peak counts are $44 \%, 11 \%$, and $63 \%$ tighter than the power spectrum constraints for $\sum m_{v}, \Omega_{m}$, and $A_{s}$, respectively. These improvements are calculated as the percentage reduction in the $95 \%$ credible regions i.e. $100 *(1-95 \%$ quantile range of joint statistics / 95\% quantile range of power spectrum).

\section{IMPACT OF BARYONS}

We use the BAHAMAS and Osato15 simulation suites to explore the impact of baryonic effects on minimum counts and their parameter constraints, using one source redshift ${ }^{5} z_{s}=1.0$. While our paper focuses on minimum counts, we also show results on peak counts. All previous works studying baryonic effects on peaks either remained at the observable level, or propagated to cosmological constraints but used only a simple Fisher formalism. We present the first study using a large set of cosmologies and full likelihood analysis.

In Fig. 6 we show the fractional difference between the BAHAMAS fiducial AGN model and the corresponding dark matter-only simulations. To see the physics more transparently, we show the effects without the galaxy shape noise. The main effect of baryons on the power spectrum is to suppress power at $\ell \gtrsim$ a few $\times 100$, consistent with results from previous work (e.g. Gouin et al. 2019). Baryonic effects suppress positive and deep negative minima, while boosting the $\kappa \approx 0$ minima. Paillas et al. (2017) found evidence in the EAGLE hydrodynamical simulations that baryonic processes

5 The effect of using tomography for minimum counts is similar to the effect on peaks (see e.g. Li et al. 2019), which can be seen by comparing the constraints in Figs. 5 and 8. can reduce the number of voids and produce less empty voids, both of which could be relevant to our findings. When noise is added, we see similar effects (see Fig. 3).

We also compare the effects of baryons to those from changing cosmological parameters in Fig. 6. One promising feature is that the fractional effects on all three observables are different for baryons than for cosmological parameters investigated here. However, effects of baryons rely heavily on subgrid models in hydrodynamical simulations, which remain somewhat uncertain (Springel et al. 2018). This is demonstrated in Fig. 7 where we show the fractional effect of baryons for the two other AGN feedback models in the BAHAMAS simulations and the "FE" model from Osato15. However, we note that BAHAMAS simulations are calibrated to match some key observables, such as the present-day baryon content of massive systems, the hot gas mass fraction-halo mass relation of galaxy groups and clusters, as well as the amplitude of the black hole mass-stellar mass relation. Hence we expect them to be more realistic and use them to study the impact of baryons on cosmological constraints. Further, the BAHAMAS low and high AGN models were constructed to capture the upper and lower bounds of the observed group and cluster gas fractions, and hence we expect them to represent the theoretical uncertainties in the effects of baryons, making them ideal to study the impact of baryons on cosmological constraints.

Next, we use the BAHAMAS simulations to model the impact of baryons on cosmological constraints. We make the assumption that the fractional effect of baryonic processes is independent of cosmology, which has been shown to be true for the power spectrum within a few percent (Mead et al. 2015, 2016; Mummery et al. 2017; van Daalen et al. 2019; Stafford et al. 2019). To include baryonic effects, we introduce into our emulator a new parameter $A_{\text {baryon, }}$, which linearly interpolates the fractional effect of baryons between no baryonic effects $\left(A_{\text {baryon }}=0\right)$ to the BAHAMAS high-AGN model $A_{\text {baryon }}=3$, with $A_{\text {baryon }}=2$ for the fiducial model and $A_{\text {baryon }}=1$ for the low-AGN model. We then jointly fit $A_{\text {baryon }}$ together with the cosmological parameters.

We show our results with baryons for lensing minima in Fig. 8 and lensing peaks in Fig. 9. We compare three cases: (1) "dark matter only" simulations, (2) "hydro sims: baryons not modeled": hydrodynamical simulations as the observable, but fitted to dark matter-only models, i.e. using an emulator with only three cosmo- 


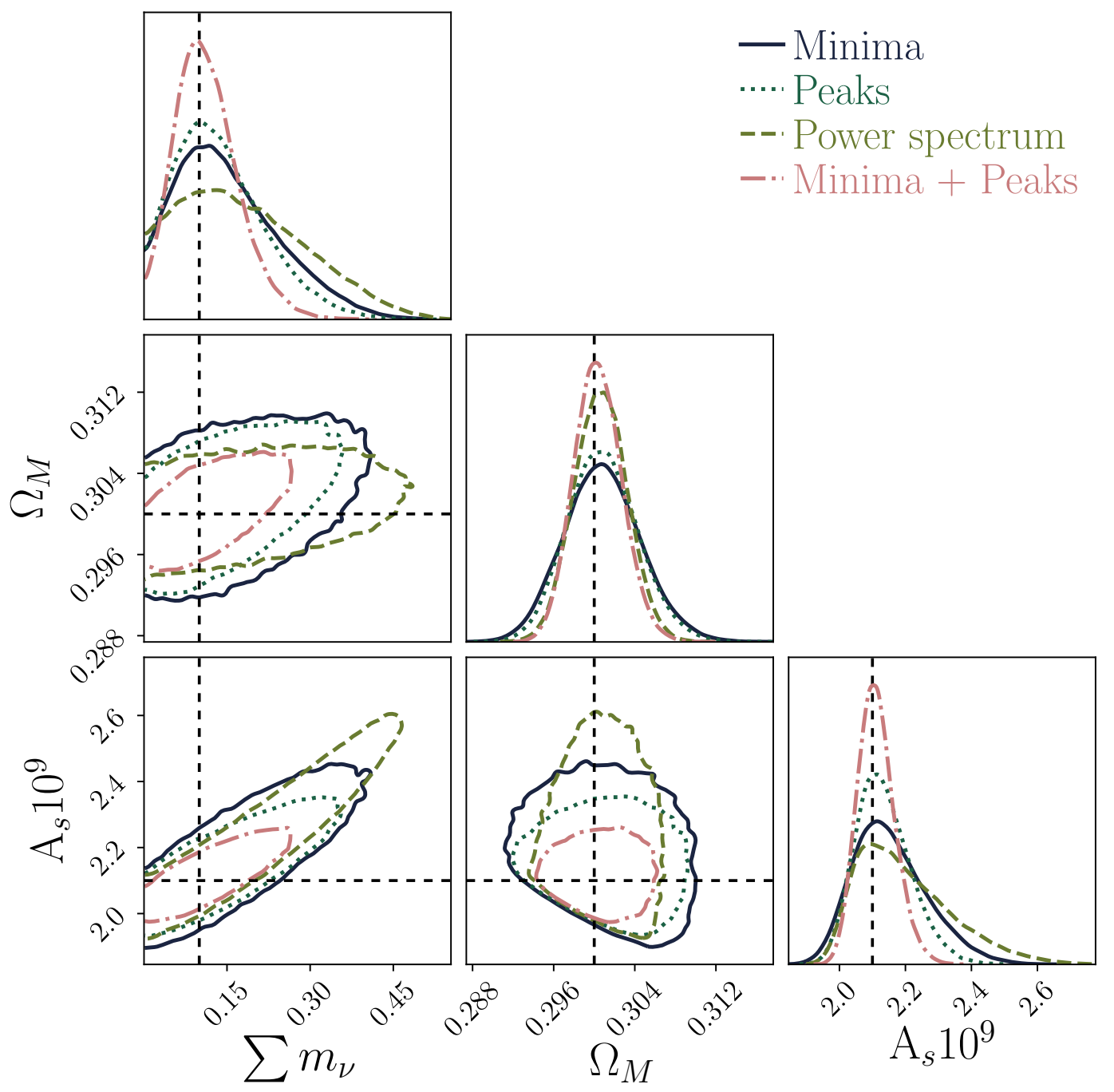

Figure 5. 95\% credible regions on $\sum m_{v}, \Omega_{m}$, and $A_{s}$ from the lensing minimum counts, peak counts, and power spectrum, and the combination of minimum and peak counts. The convergence maps are smoothed with a 2 arcmin Gaussian filter. We use 5 tomographic source redshifts and assume galaxy noises and sky coverage for an LSST-like experiment.
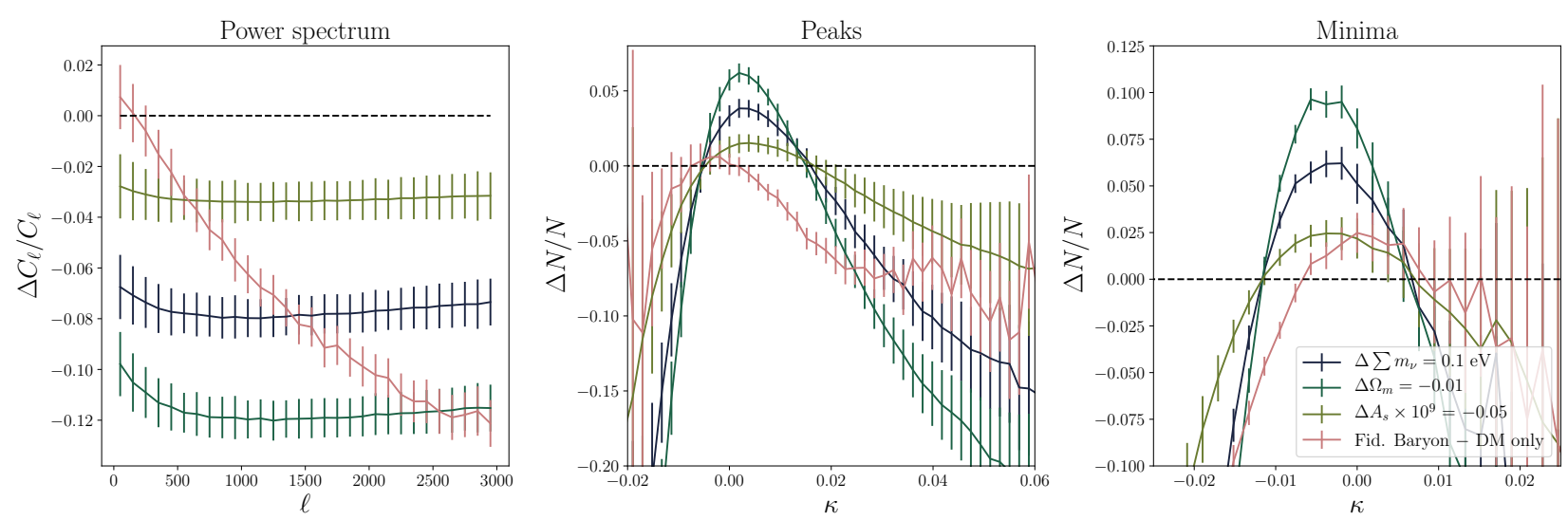

Figure 6. The fractional difference in the power spectrum (left), peak counts (center), and minimum counts (right) due to variation of three cosmological parameters $\left(\sum m_{v}, \Omega_{m}, A_{s}\right)$ and baryons without galaxy shape noise, for a single source redshift $z_{s}=1$. For the baryonic effects, we use the fiducial AGN feedback model from the BAHAMAS simulations. 

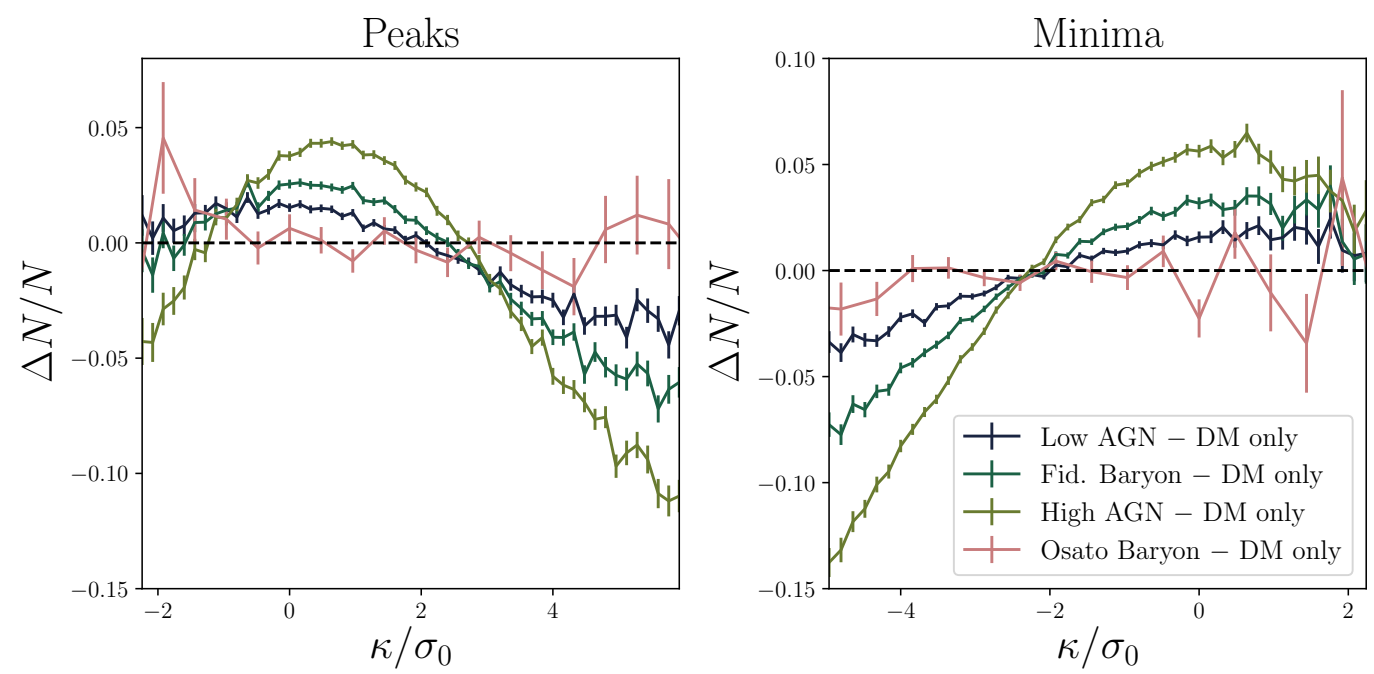

Figure 7. The fractional difference in minimum counts and peak counts due to different feedback implementations and strengths. The error bars represent the uncertainties due to the finite number of simulations; thus are given by the measured variance in the simulations $\sigma_{\text {counts }}^{\text {measured }}$ divided by the square root of the number of simulations $\sqrt{N_{\text {sim }}}$.

logical parameters $\left(\sum m_{v}, \Omega_{m}, A_{s}\right)$. This method would interpret baryonic effects as biases in cosmological parameters, and (3) "hydro sims: baryons marginalized": hydrodynamical simulations with models that include baryonic effects with four parameters $\left(\sum m_{v}\right.$, $\left.\Omega_{m}, A_{s}, A_{\text {baryon }}\right)$.

Without modeling baryons, cosmological constraints from lensing minima have mild biases $\approx 0.5 \sigma$ from the true values. In contrast, we see significant biases using peak counts, at more than $4 \sigma$ from the true values. This implies that minimum counts are a more robust statistic against baryonic effects than the peak counts. However, when we marginalize the baryonic effects (case 3), the biases vanish for both minimum counts and peak counts, implying that the effects of baryons are sufficiently distinct from the cosmological parameters and we can mitigate them by including baryons in our model.

\section{CONCLUSIONS}

We study the cosmological constraints from weak lensing minima, a simple statistic complementary to the lensing power spectrum and peak counts. Our analysis used realistic galaxy redshift distribution and shape noise as expected from LSST. We find that lensing minima contain non-Gaussian information, and provide tighter constraints than the power spectrum. Lensing minima alone are slightly less constraining than the peaks. However, when the two are combined, they produce significantly tighter constraints than the power spectrum, by $44 \%, 11 \%$, and $63 \%$ on $\sum m_{v}, \Omega_{m}$, and $A_{s}$, respectively. Our results show that lensing minima are a promising probe for upcoming cosmological experiments.

We use hydrodynamical simulations to study the effects of baryons on lensing minima. We find that baryonic processes result in a reduced number of deep (very negative $\kappa$ ) and high, positive $\kappa$ minima, while enhancing the number of shallower $(\kappa \approx 0)$ minima. We find that the baryonic effects, as modeled in the hydrodynamical simulations BAHAMAS, have little impact on minimum counts ( $\approx 0.5 \sigma$ biases), but can induce large $(\gtrsim 4 \sigma)$ biases in peak counts analysis. By extending our emulator to include baryonic processes and marginalize them, we recover the correct cosmology without losing much constraining power for both the minimum counts and peak counts. Our results emphasize the importance of modeling baryonic effects for future lensing surveys, and suggest that lensing minima can be a useful tool to mitigate the biases induced by baryons.

Our work is the first step to investigate the cosmological constraints from lensing minima and the effects of baryons on them. Future work should study other systematics, such as the intrinsic alignments of galaxies, photometric redshifts, multiplicative biases in shape measurements and the impact of masking. As we used a simple linear model to capture the baryonic effects, more accurate modeling of baryons will be beneficial, such as a more general parameterization (Harnois-Déraps et al. 2015; Schneider et al. 2019) or via a Principal Component Analysis (Eifler et al. 2015; Huang et al. 2019).

\section{ACKNOWLEDGEMENTS}

We thank Cora Uhlemann, Oliver Friedrich, Daan Meerburg, and Patrick de Perio for helpful discussions. IGM thanks Joop Schaye for his contributions to the BAHAMAS project. We are grateful to the referee for their useful comments. This work is supported by an NSF Astronomy and Astrophysics Postdoctoral Fellowship (to JL) under award AST-1602663. We acknowledge support from the WFIRST project. WRC acknowledges support from the UK Science and Technology Facilities Council (grant number ST/N000927/1). This project has received funding from the European Research Council (ERC) under the European Union's Horizon 2020 research and innovation programme (grant agreement No 769130). KO is supported by Japan Society for the Promotion of Science (JSPS) Overseas Research Fellowships. We thank New Mexico State University (USA) and Instituto de Astrofisica de Andalucia CSIC (Spain) for hosting the Skies \& Universes site for cosmological simulation products. This work used resources of the National Energy Research Scientific Computing Center (NERSC), a U.S. Department of Energy Office of Science User Facility operated under Contract No. DE-AC02- 


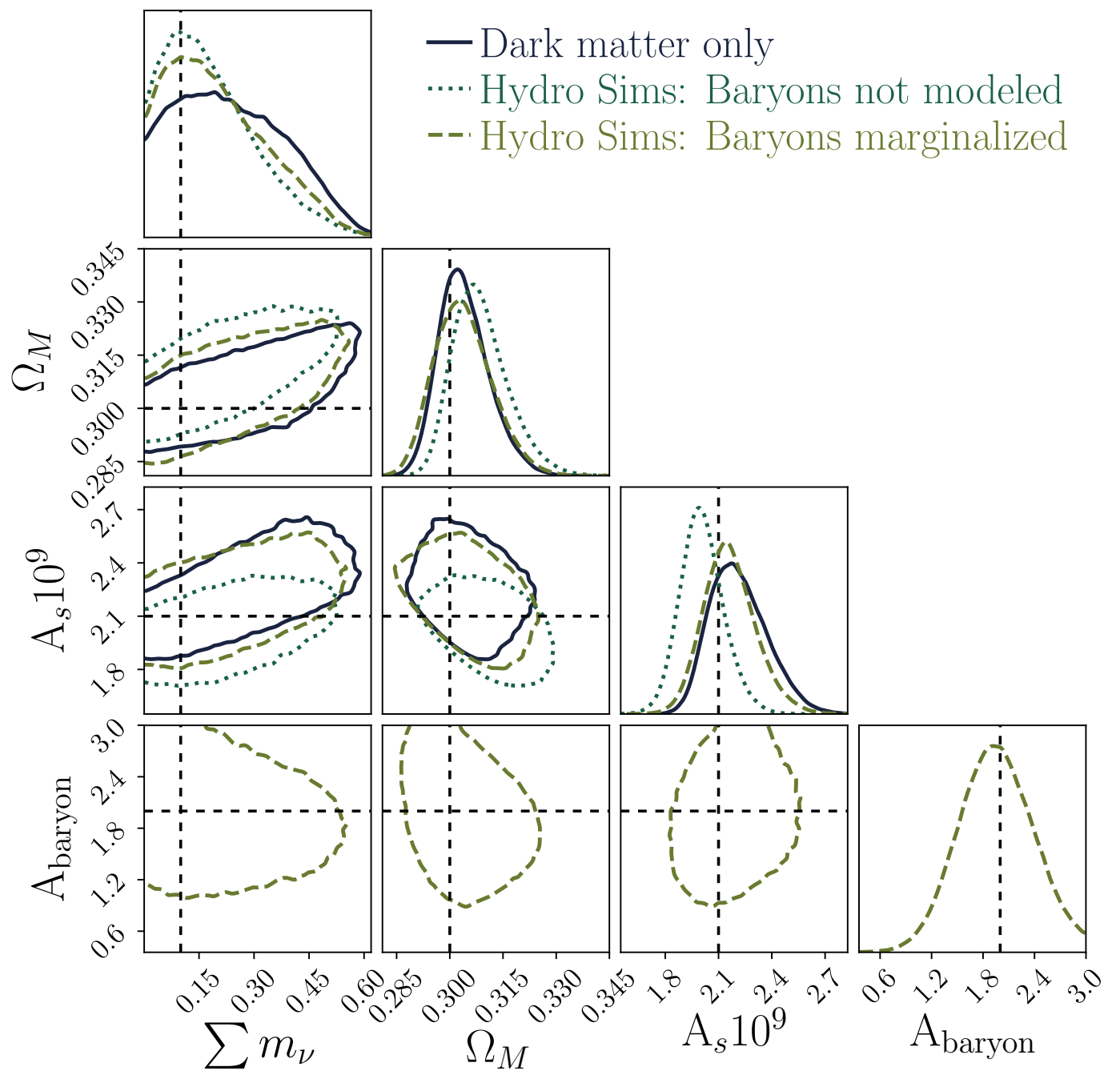

Figure 8. 95\% credible regions on $\Omega_{m}, A_{s}, \sum m_{v}$ and $A_{\text {baryon }}$ for minimum counts, for three cases: (1) dark matter-only simulations (solid), (2) hydrodynamical simulations with dark matter-only models, which would interpret baryonic effects as biases in cosmological parameters ("hydro sims: baryons not modeled", dotted), and (3) hydrodynamical simulations with models that include baryonic effects ("hydro sims: baryons marginalized", dashed), parameterized as $A_{\text {baryon. }}$.

05CH11231. This work used the Extreme Science and Engineering Discovery Environment (XSEDE), which is supported by NSF grant ACI-1053575. The analysis is in part performed at the TIGRESS high performance computer center at Princeton University. This work used the DiRAC@ Durham facility managed by the Institute for Computational Cosmology on behalf of the STFC DiRAC HPC Facility (https://www.dirac.ac.uk). The equipment was funded by BEIS capital funding via STFC capital grants ST/K00042X/1, ST/P002293/1, ST/R002371/1 and ST/S002502/1, Durham University and STFC operations grant ST/R000832/1. DiRAC is part of the National e-Infrastructure. Numerical simulations were in part carried out on Cray XC30 at the Center for Computational Astrophysics, National Astronomical Observatory of Japan.

\section{APPENDIX A: THE EFFECT OF SMOOTHING SCALES}

In the main analysis, we used a 2 arcmin smoothing scale. Here we explore how the $95 \%$ credible regions from lensing minima and peaks change as we vary the smoothing scales (1, 2, and 5 arcmin).
In Fig. A1, we find that the constraining power degrades quickly with increasing smoothing scales for both statistics, as we start to lose small scales where non-Gaussian information is the richest. Most past works on cosmological constraints from weak lensing data used large smoothing scales ( $~ 10 \mathrm{arcmin}$ ) to avoid baryonic effects. From our main results, we find that lensing peaks can indeed be highly biased by baryonic effects and hence requires careful modeling. However, we find lensing minima somewhat insensitive to baryonic effects and hence may be a more robust tool to calibrate cosmology on small scales.

\section{APPENDIX B: COMPARISON TO GENUS}

Minkowski functionals characterize the morphology of a field and are sensitive to non-Gaussian distributions. For a two dimensional field, there are three Minkowski functionals: the area $V_{0}$, perimeter $V_{1}$, and genus $V_{2}$. Previous work by Petri et al. (2013, 2015); Marques et al. (2019) found that Minkowski functionals can offer strong 


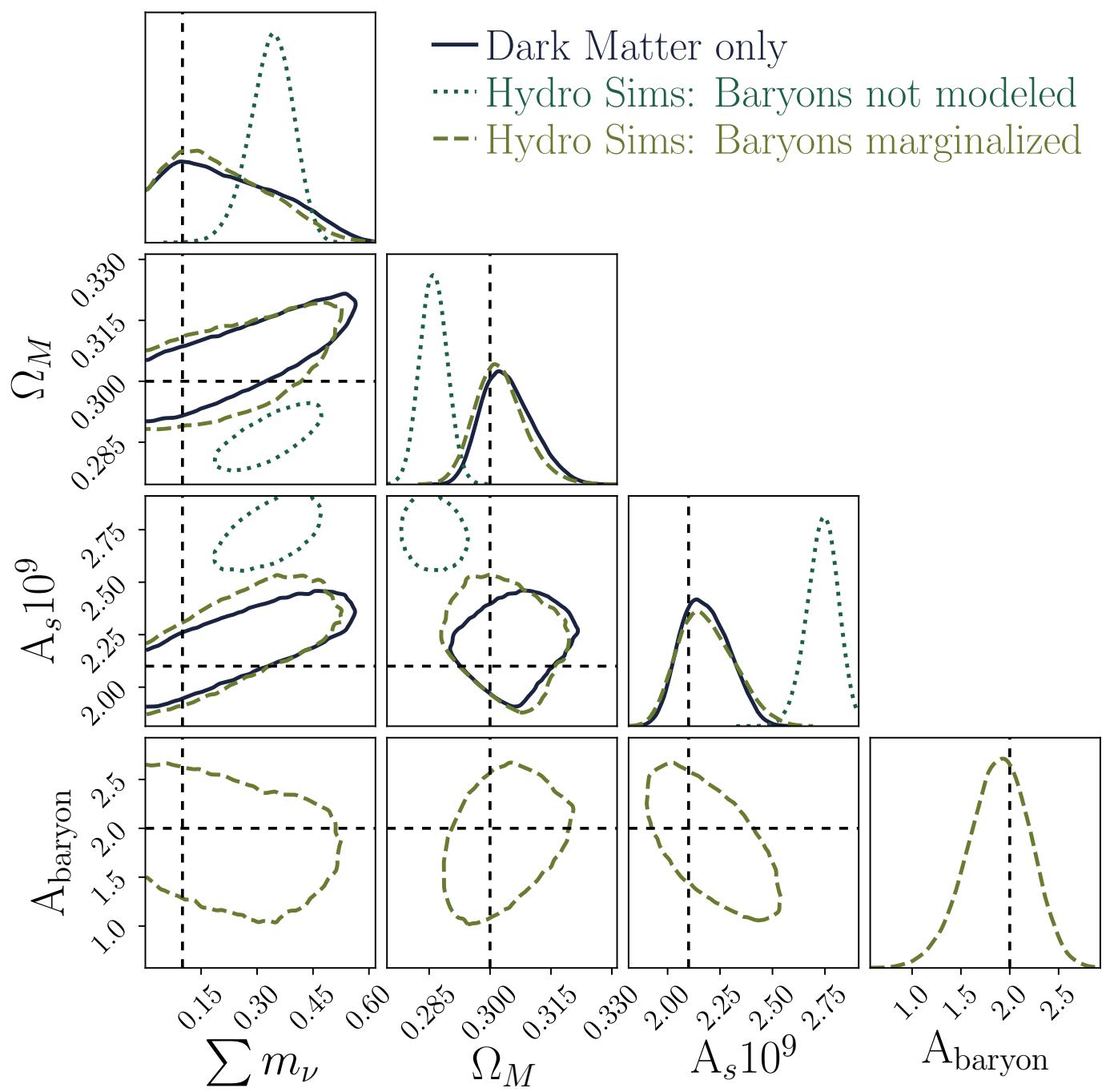

Figure 9. 95\% credible regions on $\Omega_{m}, A_{s}, \sum m_{v}$ and $A_{\text {baryon }}$ for peak counts, for three cases: (1) dark matter-only simulations (solid), (2) hydrodynamical simulations with dark matter-only models, which would interpret baryonic effects as biases in cosmological parameters ("hydro sims: baryons not modeled", dotted), and (3) hydrodynamical simulations with models that include baryonic effects ("hydro sims: baryons marginalized", dashed), parameterized as $A_{\text {baryon. }}$.

cosmological constraints. In this Appendix, we are particularly interested in the genus $V_{2}$ - the difference between the number of "holes" and the number of "islands", which we think can be closely related to the minimum counts and peaks counts in the field, respectively.

Genus is expressed as a function of the threshold $\kappa_{0}$,

$V_{2}\left(\kappa_{0}\right)=\frac{1}{2 \pi A} \int_{\partial \Sigma\left(\kappa_{0}\right)} \mathrm{d} l \mathcal{K}$,

where $A$ is the total area of the field, $\mathcal{K}$ is the curvature, $\Sigma\left(\kappa_{0}\right)$ is the excursion set of all pixels with $\kappa \geq \kappa_{0}$, and $\partial \Sigma\left(\kappa_{0}\right)$ is the boundary of the excursion set. Their connection can be explicitly verified by examining their cosmological information. In Fig. B1 we show the parameter constraints from the genus of the lensing field and the combination of lensing peaks and minima. We find that their constraints are almost identical.

\section{REFERENCES}

Ali-Haïmoud Y., Bird S., 2013, MNRAS, 428, 3375

Baker T., Clampitt J., Jain B., Trodden M., 2018, Phys. Rev. D, 98, 023511 Barreira A., Cautun M., Li B., Baugh C. M., Pascoli S., 2015, J. Cosmology Astropart. Phys., 2015, 028

Barreira A., Bose S., Li B., Llinares C., 2017, J. Cosmology Astropart. Phys., 2017, 031

Bernardeau F., van Waerbeke L., Mellier Y., 1997, A\&A, 322, 1

Bird S., Ali-Haïmoud Y., Feng Y., Liu J., 2018, MNRAS,

Bond J. R., Efstathiou G., 1987, MNRAS, 226, 655

Booth C. M., Schaye J., 2009, MNRAS, 398, 53

Clampitt J., Jain B., 2015, MNRAS, 454, 3357

Coulton W. R., Liu J., Madhavacheril M. S., Böhm V., Spergel D. N., 2019, J. Cosmology Astropart. Phys., 5, 043

DES Collaboration et al., 2017, preprint, (arXiv: 1708.01530)

Davies C. T., Cautun M., Li B., 2018, MNRAS, 480, L101

Eifler T., Krause E., Dodelson S., Zentner A. R., Hearin A. P., Gnedin N. Y., 2015, MNRAS, 454, 2451

Fong M., Choi M., Catlett V., Lee B., Peel A., Bowyer R., King L. J., McCarthy I. G., 2019, MNRAS, 488, 3340 


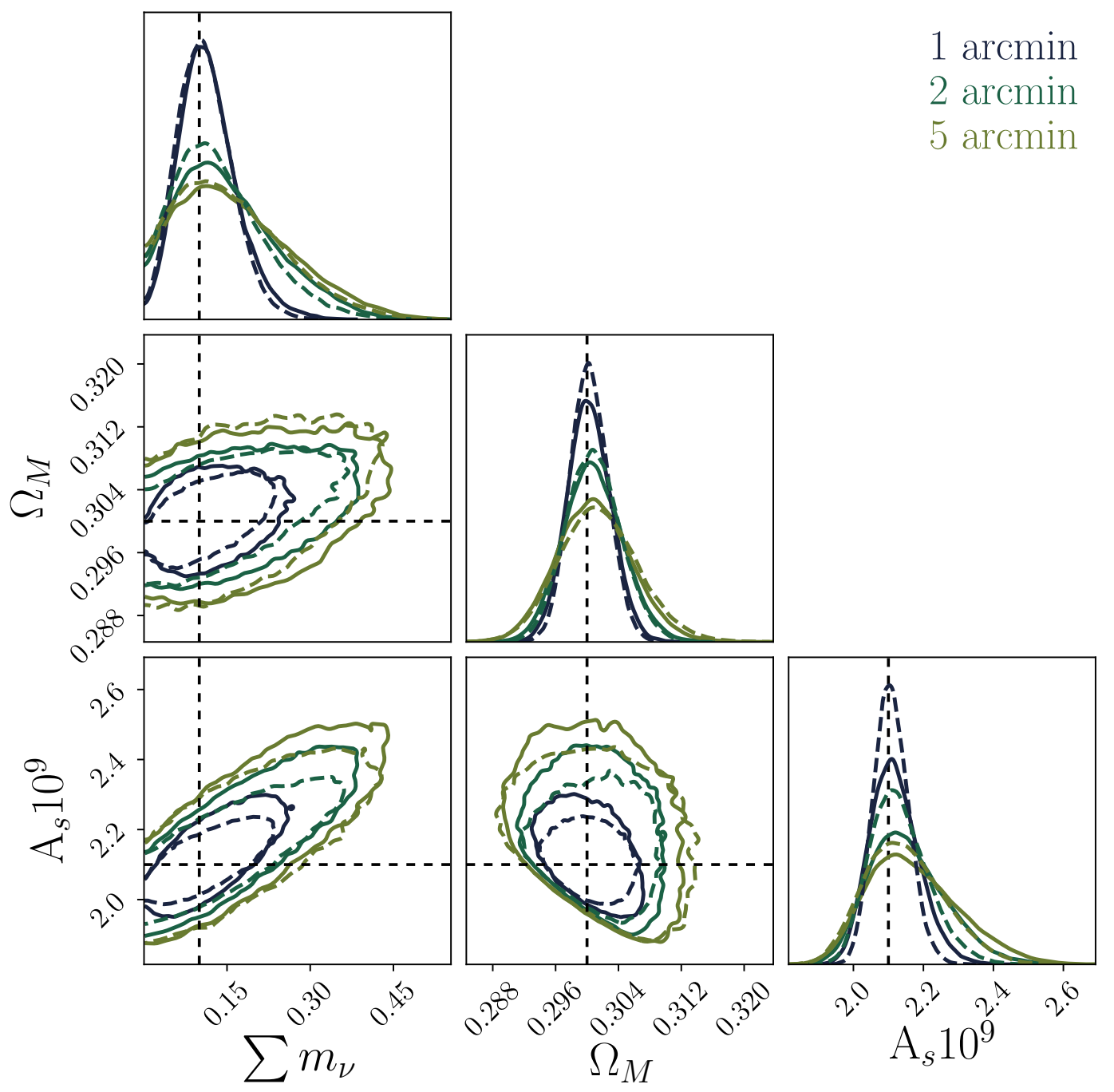

Figure A1. 95\% credible regions from minimum counts (solid lines) and peak counts (dashed lines) for three different smoothing scales, using 5 tomographic redshifts and LSST-like galaxy noise.

Foreman-Mackey D., 2016, The Journal of Open Source Software, 24 Foreman-Mackey D., Hogg D. W., Lang D., Goodman J., 2013, PASP, 125, 306

Fu L., et al., 2014, MNRAS, 441, 2725

Giblin B., et al., 2018, preprint, (arXiv: 1805.12084)

Gouin C., et al., 2019, A\&A, 626, A72

Gruen D., et al., 2016, MNRAS, 455, 3367

Harnois-Déraps J., van Waerbeke L., Viola M., Heymans C., 2015, MNRAS, 450,1212

Heitmann K., Higdon D., White M., Habib S., Williams B. J., Lawrence E., Wagner C., 2009, ApJ, 705, 156

Heitmann K., et al., 2016, ApJ, 820, 108

Heymans C., et al., 2012, MNRAS, 427, 146

Hikage C., et al., 2019, PASJ, 71, 43

Hildebrandt H., et al., 2017, MNRAS, 465, 1454

Hinshaw G., et al., 2013, ApJS, 208, 19

Hinton S. R., 2016, The Journal of Open Source Software, 1, 00045

Huang H.-J., Eifler T., Mandelbaum R., Dodelson S., 2019, MNRAS, 488, 1652

Hui L., 1999, ApJ, 519, L9

Jain B., Van Waerbeke L., 2000, ApJ, 530, L1

Kacprzak T., et al., 2016, preprint, (arXiv:1603.05040)

Kilbinger M., 2015, Reports on Progress in Physics, 78, 086901
Kilbinger M., Schneider P., 2005, A\&A, 442, 69

Kratochvil J. M., Lim E. A., Wang S., Haiman Z., May M., Huffenberger K., 2012, Phys. Rev. D, 85, 103513

Kreisch C. D., Pisani A., Carbone C., Liu J., Hawken A. J., Massara E., Spergel D. N., Wandelt B. D., 2019, MNRAS, 488, 4413

Kwan J., Bhattacharya S., Heitmann K., Habib S., 2013, ApJ, 768, 123

Kwan J., Heitmann K., Habib S., Padmanabhan N., Lawrence E., Finkel H., Frontiere N., Pope A., 2015, ApJ, 810, 35

LSST Science Collaboration et al., 2009a, preprint, (arXiv: 0912.0201)

LSST Science Collaboration et al., 2009b, arXiv e-prints, p. arXiv:0912.0201

Lesgourgues J., Pastor S., 2006, Phys. Rep., 429, 307

Li Z., Liu J., Matilla J. M. Z., Coulton W. R., 2019, Phys. Rev. D, 99, 063527

Lin C.-A., Kilbinger M., 2015a, A\&A, 576, A24

Lin C.-A., Kilbinger M., 2015b, A\&A, 583, A70

Liu X., et al., 2014, preprint, (arXiv: 1412.3683)

Liu J., Petri A., Haiman Z., Hui L., Kratochvil J. M., May M., 2015, Phys. Rev. D, 91, 063507

Liu J., Bird S., Matilla J. M. Z., Hill J. C., Haiman Z., Madhavacheril M. S., Spergel D. N., Petri A., 2018, Journal of Cosmology and Astroparticle Physics, 2018

Marian L., Smith R. E., Bernstein G. M., 2009, ApJ, 698, L33

Marian L., Smith R. E., Hilbert S., Schneider P., 2013, MNRAS, 432, 1338 


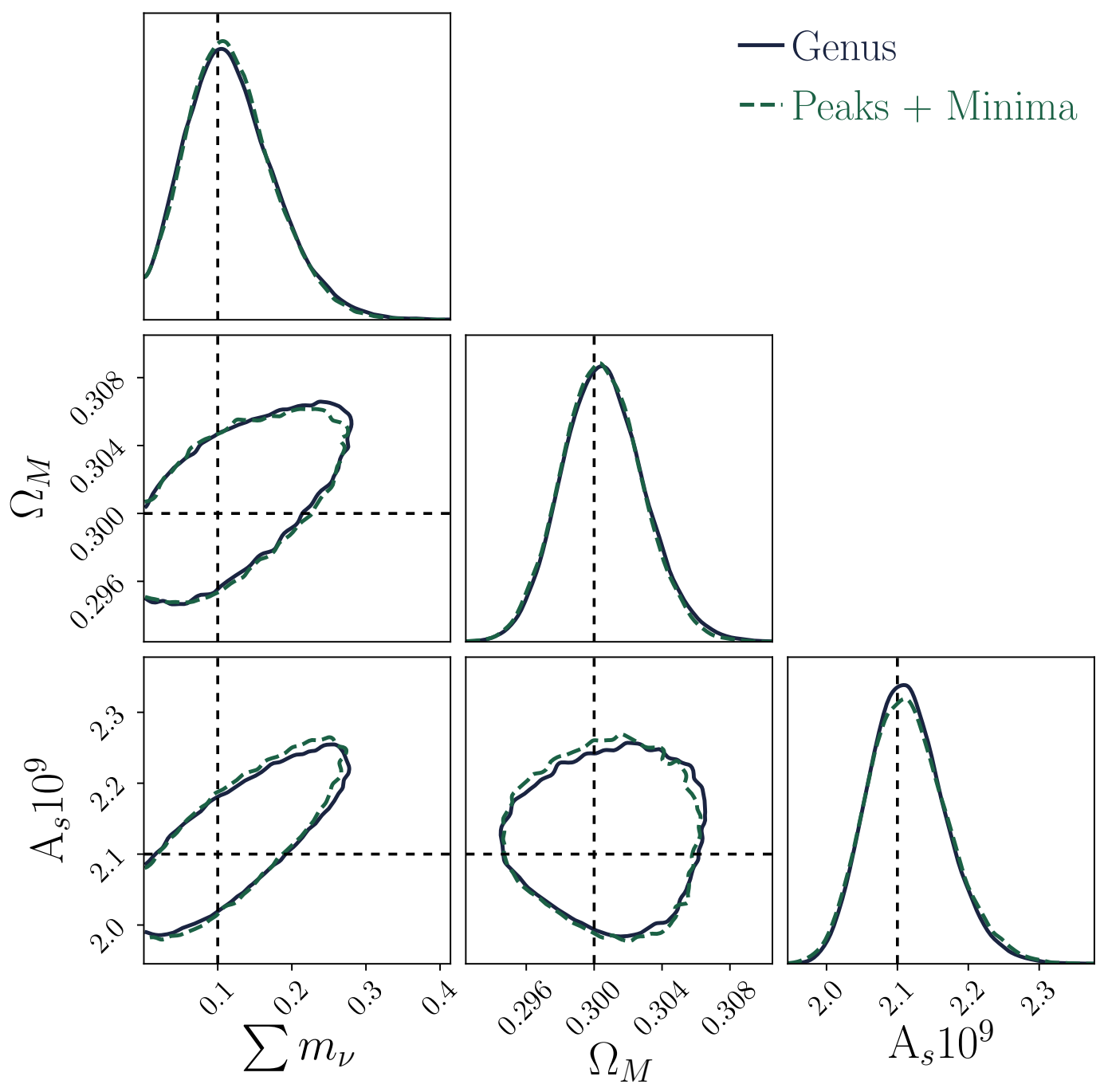

Figure B1. 95\% credible regions from the combination of minimum counts and peak counts (dashed) and that from the third Minkowski functional the genus (solid), using 5 tomographic redshifts and LSST-like galaxy noise.

Marques G. A., Liu J., Zorrilla Matilla J. M., Haiman Z., Bernui A., Novaes C. P., 2019, J. Cosmology Astropart. Phys., 2019, 019

Martinet N., et al., 2018, MNRAS, 474, 712

Massara E., Villaescusa-Navarro F., Viel M., Sutter P. M., 2015, J. Cosmology Astropart. Phys., 2015, 018

Maturi M., Angrick C., Pace F., Bartelmann M., 2010, A\&A, 519, A23

McCarthy I. G., Schaye J., Bird S., Le Brun A. M. C., 2017, MNRAS, 465, 2936

McCarthy I. G., Bird S., Schaye J., Harnois-Deraps J., Font A. S., van Waerbeke L., 2018, MNRAS, 476, 2999

McClintock T., et al., 2019, ApJ, 872, 53

Mead A. J., Peacock J. A., Heymans C., Joudaki S., Heavens A. F., 2015, MNRAS, 454, 1958

Mead A. J., Heymans C., Lombriser L., Peacock J. A., Steele O. I., Winther H. A., 2016, MNRAS, 459, 1468

Melchior P., Sutter P. M., Sheldon E. S., Krause E., Wandelt B. D., 2014, MNRAS, 440, 2922

Mummery B. O., McCarthy I. G., Bird S., Schaye J., 2017, MNRAS, 471, 227

Nelson D., et al., 2019, Computational Astrophysics and Cosmology, 6, 2

Nishimichi T., et al., 2018, arXiv e-prints, p. arXiv:1811.09504

Okamoto T., Shimizu I., Yoshida N., 2014, PASJ, 66, 70

Osato K., Shirasaki M., Yoshida N., 2015, ApJ, 806, 186
Paillas E., Lagos C. D. P., Padilla N., Tissera P., Helly J., Schaller M., 2017, MNRAS, 470, 4434

Paillas E., Cautun M., Li B., Cai Y.-C., Padilla N., Armijo J., Bose S., 2019, MNRAS, 484, 1149

Peel A., Pettorino V., Giocoli C., Starck J.-L., Baldi M., 2018, preprint, (arXiv: 1805.05146)

Petri A., 2016, Astronomy and Computing, 17, 73

Petri A., Haiman Z., Hui L., May M., Kratochvil J. M., 2013, Phys. Rev. D, 88,123002

Petri A., Liu J., Haiman Z., May M., Hui L., Kratochvil J. M., 2015, Phys. Rev. D, 91, 103511

Sánchez C., et al., 2017, MNRAS, 465, 746

Schaye J., et al., 2010, MNRAS, 402, 1536

Schaye J., et al., 2015, MNRAS, 446, 521

Schneider A., Teyssier R., Stadel J., Chisari N. E., Le Brun A. M. C., Amara A., Refregier A., 2019, J. Cosmology Astropart. Phys., 2019, 020

Shan H., et al., 2018, MNRAS, 474, 1116

Shirasaki M., Yoshida N., 2014, ApJ, 786, 43

Springel V., et al., 2018, MNRAS, 475, 676

Stafford S. G., McCarthy I. G., Crain R. A., Salcido J., Schaye J., Font A. S., Kwan J., Pfeifer S., 2019, arXiv e-prints, p. arXiv:1907.09497

Takada M., Jain B., 2002, MNRAS, 337, 875

Takada M., Jain B., 2003, MNRAS, 344, 857 
Vafaei S., Lu T., van Waerbeke L., Semboloni E., Heymans C., Pen U.-L., 2010, Astroparticle Physics, 32, 340

Van Waerbeke L., Hamana T., Scoccimarro R., Colombi S., Bernardeau F., 2001, MNRAS, 322, 918

Weiss A. J., Schneider A., Sgier R., Kacprzak T., Amara A., Refregier A., 2019, arXiv e-prints, p. arXiv:1905.11636

Yang X., Kratochvil J. M., Wang S., Lim E. A., Haiman Z., May M., 2011, Phys. Rev. D, 84, 043529

Yang X., Kratochvil J. M., Huffenberger K., Haiman Z., May M., 2013, Phys. Rev. D, 87, 023511

Zaldarriaga M., Scoccimarro R., 2003, ApJ, 584, 559

van Daalen M. P., Schaye J., Booth C. M., Dalla Vecchia C., 2011, MNRAS, 415,3649

van Daalen M. P., McCarthy I. G., Schaye J., 2019, arXiv e-prints, p. arXiv: 1906.00968

This paper has been typeset from a $\mathrm{T}_{\mathrm{E}} \mathrm{X} / \mathrm{L} \mathrm{A} \mathrm{T}_{\mathrm{E}} \mathrm{X}$ file prepared by the author. 